\title{
Dissolved Oxygen Measurement in Seawater and Sensor Calibration Method
}

\author{
Shun $\mathrm{Gao}^{1}$, Yingying Zhang ${ }^{1, *}$, Xiandong Feng ${ }^{1}$, Da Yuan $^{1}$, Bingwei $\mathrm{Wu}^{1}$ and Yunyan \\ Zhang $^{1}$ \\ ${ }^{1}$ Qilu University of Technology (Shandong Academy of Sciences) Institute of Oceanographic \\ Instrumentation, Shandong Provincial Key Laboratory of Ocean Environmental Monitoring \\ Technology, National Engineering and Technological Research Center of Marine Monitoring \\ Equipment, Qingdao 266061, China
}

\begin{abstract}
The development and key calibration methods of the sensors for the measurement and automatic monitoring of dissolved oxygen in seawater have always been the research focus in the field of Marine monitoring technology. This paper summarizes the measurement methods of dissolved oxygen in seawater, the working principle and advantages and disadvantages of different dissolved oxygen sensors, and the research on calibration methods of mainstream optical dissolved oxygen sensors. Especially for optical dissolved oxygen sensors with better stability, longer service life and stronger anti-interference ability, several laboratory calibration methods which have been developed and applied, as well as in situ calibration methods which are still in the research stage are analyzed.
\end{abstract}

\section{Introduction}

Dissolved oxygen in seawater is an important indicator of Marine environmental monitoring and has important research significance. Among the measurement methods of dissolved oxygen in seawater, Winkler iodine method, Clark electrode method and optical method have become the main measurement methods that have been applied with their unique advantages. Based on the above three main measurement methods, applications, advantages and disadvantages of dissolved oxygen in seawater, this article focuses on the analysis of the sensor calibration method, which is the core of the measurement accuracy of the automatic measurement sensor, the type and measurement principle and the relationship between them, especially at present The in-situ calibration method of the optical dissolved oxygen sensor that is still in the research stage is analyzed and described, in order to provide a reference for the research of dissolved oxygen measurement method and sensor application.

\footnotetext{
*Corresponding author:Gaoshun_qlu@163.com
} 


\section{Method for measurement of dissolved oxygen in seawater}

\subsection{Winkler iodimetry}

Winkler iodimetry uses the reaction relationship between manganese ions, iodine reagent and dissolved oxygen to calculate the concentration of dissolved oxygen.

The concentration of dissolved oxygen measured by this method with standard solution has high reproducibility and few influencing factors. However, from the point of view of the measurement process, sampling and titration may cause subjective errors, the measurement operation process is complicated and requires professionals, and more importantly, it cannot meet the requirements of real-time measurement and continuous measurement. But Winkler's iodimetry remains the standard laboratory method for measuring dissolved oxygen in water samples, and its data are used as a reference for sensor calibration.

\subsection{Clark electrode method}

\subsubsection{Summary.}

The Clark electrode method uses the current generated by electrons in the REDOX reaction to calculate the dissolved oxygen content. Clark electrode sensor has fast measurement speed, simple operation, and can avoid the interference of impurity particles interacting with iodine. However, there are still many drawbacks to this method that affect practical application: Working electrode and film are easily aged; Other solvents and gases in water and magnetic fields will affect the measurement accuracy of the sensor; Fault in sensor measurement data during replacement of damaged film; Oxidation-reduction reaction will consume dissolved oxygen in the water sample, so the diffusion rate of dissolved oxygen in the water sample will affect the measurement accuracy of the sensor. In order to realize the long-term and continuous monitoring of dissolved oxygen, it is necessary to develop related technologies to overcome the above shortcomings.

\subsubsection{Research progress.}

Shitanda[1] used $3 \mu \mathrm{m}$ thick PDMS sensing film to increase the detection limit to 8.41 $\mu \mathrm{g} \cdot \mathrm{L}^{-1}$, and the linear correlation coefficient between the correction curve and the concentration of dissolved oxygen was 0.996. Martin[2] developed a Ni-Saran polymer film and coated it on a platinum electrode, achieving a detection range of 3.95-9.2 $\mathrm{mg} \cdot \mathrm{L}^{-1}$ with a detection limit of $0.71 \mathrm{mg} \cdot \mathrm{L}^{-1}$. Li[3] investigated A simple and efficient sensor for the synthesis of A reduced $\mathrm{GO} /$ silver nanoparticle composite modified forage carbon electrode based on electrical displacement, with A sensitivity of $0.205 \mu \mathrm{A} \cdot \mu \mathrm{M}^{-1}$ and excellent repeatability, reproducibility and anti-interference capability. Moya[4] developed A fully ink-jet dissolved oxygen sensor with A good linear response in the range of $0-8 \mathrm{mg} \cdot \mathrm{L}^{-1}$ with A detection limit of $0.11 \mathrm{mg} \cdot \mathrm{L}^{-1}$ and A sensitivity of $0.03 \mu \mathrm{A} \cdot \mathrm{L} \cdot \mathrm{mg}^{-1}$. Lee[5] replaced the liquid electrolyte with a solid electrolyte and did not require an oxygen permeable membrane, allowing continuous measurement of the total dissolved oxygen in water without changing the sensing membrane. 
Table 1. Comparison of different methods. Among them, Winkler represents Winkler Iodimetry, Clark Electrode Method, and Optical represents Optical Fluorescence Quenching.

\begin{tabular}{ccccccc}
\hline & $\begin{array}{c}\text { On-line } \\
\text { inspection }\end{array}$ & $\begin{array}{c}\text { Response } \\
\text { time }\end{array}$ & $\begin{array}{c}\text { Oxygen } \\
\text { consumption }\end{array}$ & Reagent & $\begin{array}{c}\text { Breathable } \\
\text { membrane }\end{array}$ & $\begin{array}{c}\text { Regular } \\
\text { calibration }\end{array}$ \\
\cline { 2 - 7 } Winkler & $/$ & $/$ & $/$ & $\sqrt{ }$ & $/$ & $/$ \\
Clark & $\sqrt{ }$ & $\sim 30 \mathrm{~s}$ & $\sqrt{ }$ & $/$ & $\sqrt{ }$ & $\sqrt{ }$ \\
Optical & $\sqrt{ }$ & $<8 \mathrm{~s}$ & $/$ & $/$ & $\sqrt{ }$ & $\sqrt{ }$ \\
\hline
\end{tabular}

\subsection{Optical fluorescence quenching}

\subsubsection{Summary.}

Optical dissolved oxygen sensor based on optical fluorescence quenching principle has gradually become the most concerned measurement method in the international research and development of seawater dissolved oxygen automatic monitoring.

Relatively speaking, the optical dissolved oxygen sensor is more suitable for on-site automation and long-term continuous monitoring of the ocean, and is gradually applied to a variety of autonomous ocean observation platforms. Although the optical dissolved oxygen sensor has better optical stability, longer service life and stronger interference ability, the data drift phenomenon and drift law of the optical dissolved oxygen sensor have not been clearly determined, so the calibration sensor drift is an important problem that needs to be solved in sensor development and application at present.

\subsubsection{Research progress.}

In order to further improve the measurement accuracy, Campo[6] adopted the optical fiber coupled LED light source and used the phase-locked amplifier to measure the phase lag and retrieve the oxygen concentration. The measurement accuracy reached $0.02 \mathrm{mg} \cdot \mathrm{L}^{-1}$. Shortreed[7] overcame the leakage problem of fluorescent indicator and improved the detection limit of the system to $1 \times 10^{-7} \mathrm{~mol}^{-} \mathrm{L}^{-1}$. Xavier[8] made a high-performance membrane by strictly controlling the conditions of the production of the sensing membrane, and the sensor accuracy reached $0.2 \mathrm{mg} \cdot \mathrm{L}^{-1}$. Wolfbeis[9] fixed the fluorescent indicator in porous glass to build a highly sensitive probe that reduced the reaction time to less than 5 seconds. Zaitsev[10] developed a dissolved oxygen sensor that can achieve stable measurement at $5-35^{\circ} \mathrm{C}$.

The study of China started late, but it has made some achievements. Combined with the current advanced research results of battery and communication technology, the internal power supply and underwater wireless communication are studied. It is suggested to improve the sensor integration, modularize the sensor design and production, and enhance the independent monitoring ability of the sensor.
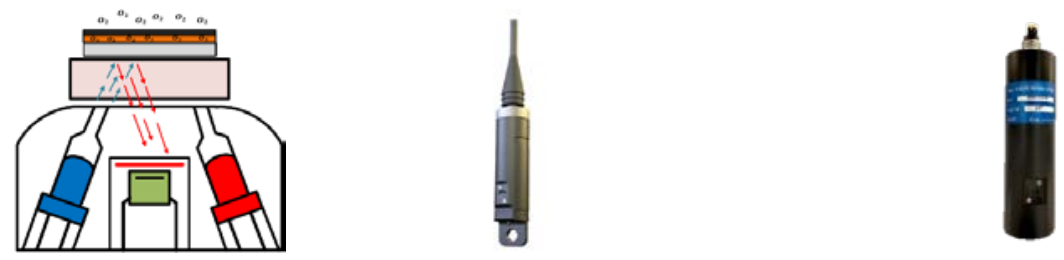

Fig. 1. Diagram of Optical Sensor 


\section{Calibration method of optical dissolved oxygen sensor}

Long-term monitoring of Marine ecological environment puts forward higher requirements for the dissolved oxygen sensor. The stability and accuracy of monitoring data become the main research direction of the dissolved oxygen sensor. During the measurement process of optical dissolved oxygen sensor, data drift will occur due to unavoidable reasons such as membrane decomposition, so regular calibration must be conducted to ensure data quality.

\subsection{Laboratory calibration method}

Before that, the computational models and calibration procedures of laboratory calibration methods have been gradually developed and perfected, and the laboratory is usually able to meet the necessary conditions for accurate calibration of optical dissolved oxygen sensors. Therefore, the laboratory calibration method has always been one of the most extensive calibration methods for the development, production and use of optical dissolved oxygen sensors.

\subsubsection{Quaternary polynomial method.}

Quaternary polynomial method is one of the earliest laboratory calibration methods studied and applied. The principle is to establish a calibration relationship between the phase and the concentration of $\mathrm{O}_{2}$. In general, although this method can provide us with fairly accurate calibration results, However, the calibration method needs to use a large number of experimental data to obtain the correlation coefficient in the calibration relationship, and the quality of the experimental data is relatively high, leading to the harsh conditions of the calibration experiment and cumbersome operation, so the calibration period is long. The calibration model of this method is empirically established, not the theoretical relationship of the measurement method.

\subsubsection{Stern - Volmer method.}

According to Uchida[11] in 2008, based on the theoretical relationship between oxygen concentration and luminescence decay time, a new model was proposed to calibrate the dissolved oxygen concentration from the phase shift data of the dissolved oxygen sensor.

On the one hand, this method describes the characteristics of the sensor from the theoretical relationship, on the other hand, it simplifies the steps and complexity of calibration experiments, and at the same time, it can obtain ideal calibration results. Therefore, it has become the most widely used laboratory calibration method at present.

\subsection{In Situ calibration method}

The data drift phenomenon of optical dissolved oxygen sensor in the field monitoring process leads to the accuracy and reliability of dissolved oxygen data decreased greatly. To improve sensor performance or develop calibration technology, make breakthrough progress in detection accuracy, stability and other aspects, and realize continuous and accurate measurement of dissolved oxygen monitoring is an urgent problem to be solved. 


\subsubsection{Two-point calibration method.}

The two-point method can be used for both laboratory and field calibration. At constant temperature, the optical dissolved oxygen sensor was immersed in samples with 0 and $100 \%$ oxygen saturation respectively to collect data, and the two-point calibration formula and calibration coefficient were calculated. Compared with other calibration methods, the two-point method has the advantages of extremely short calibration cycle, simple operation process, less reagent use and high operability, but poor calibration accuracy. It is suitable for data drift caused by low requirements for measurement accuracy or adhesion of foreign bodies in the sensing film. However, this calibration method provides a good idea for in-situ calibration: configure the standard solution and put it on the sensor to complete in-situ calibration, thus reducing the maintenance cost of the sensor.

\subsubsection{Climatological data calibration methods.}

Climatological data calibration method is an in-situ calibration method proposed by Takeshita[12] This method uses the World Ocean Atlas WOA to calibrate optical dissolved oxygen sensors. Dissolved oxygen was measured by Winkler iodimetry to ensure the accuracy of the data.

After decades of data accumulation, the dissolved oxygen data provided by WOA is an effective reference, but the results of the in-situ calibration method show that this method is suitable for the calibration of optical dissolved oxygen sensors that cannot be completely exposed to air. It is suggested to supplement the accurate data of dissolved oxygen obtained with WOA verification, and to establish a three-dimensional observation database from bottom to surface by using observation technologies such as Argo, Glider and surface buoys in conjunction with the development of current communication network technology. To achieve high resolution and high precision data of dissolved oxygen is the main direction of future development using WOA calibration sensors.

\subsubsection{Calibration methods for air measurements.}

To perform the calibration, the optical dissolved oxygen sensor is completely exposed to the air and the dissolved oxygen concentration is read by the external air pressure and the sensor calibrated assuming 100\% oxygen saturation. Based on the different responses of the optical dissolved oxygen sensor in different media, the calibration of the optical dissolved oxygen sensor is completed.

Air measurement calibration method in continuous monitoring, in situ calibration, saturation air calibration model and other technical innovations, breakthrough laboratory calibration limits. Calibration for the drift or aging of the dissolved oxygen sensor in the long-term monitoring process can effectively enhance the accuracy and continuity of the observation data. However, the application and industrialization of air measurement calibration method is an urgent problem to be solved.

\subsubsection{The research progress.}

Kortzinger[13] used data from NCEP-NCAR to predict oxygen values at the sensor deployment site, providing a means of controlling quality by directly measuring data, and providing a means of identifying drift problems. D 'Asaro[14] accurately explained the matching of the calibration equation with the physical properties of the optical dissolved oxygen sensor and determined that the drift in oxygen was nonlinear and decay exponent in time. Takeshita[12]. compared the profile buoy data with the world ocean atlas and verified 
the feasibility of the calibration method, which is of great significance for promoting the application of profile buoy data sets. Bushinsky[15] studied the feasibility of in-situ calibration with optical dissolved oxygen sensors on mooring equipment, which provided a reliable reference for other ocean observation platforms. Johnson[16] collected oxygen data from calibrated AANDEA sensors on profiling buoys and compared it with Winkler's iodimetry and the Marine Atlas of the World, and found a 10-fold reduction in error. Nicholson and Feen[17] performed air calibration on the optical dissolved oxygen sensor on the glider, and the calibrated data were consistent with Winkler's measurements.
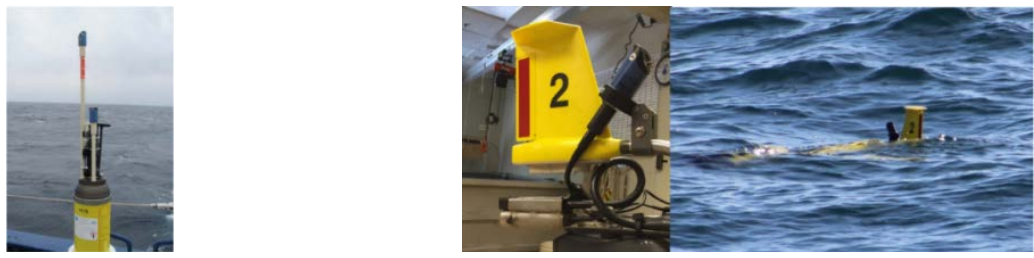

Fig. 2. Sensors on the autonomous observation platform

\section{Conclusion and prospect}

For seawater dissolved oxygen, this paper summarized and analyzed the measurement methods, monitoring sensors and key calibration methods, focusing on the summary and analysis of optical dissolved oxygen sensor calibration methods and applications.

In terms of in situ calibration methods, air measurement, as a new method, should be verified under more complex and extensive ocean conditions. Moreover, there are still uncertainties in the accuracy and drift of air calibration when oxygen concentration is low, further study is needed. Secondly, the actual in-situ calibration process found that the sensor in the "water-air continuous measurement mode" may not observe the oxygen content in the air, sensors may be affected by water during air exposure, leading to an early end to the "air measurement" and increasing uncertainty in the measurement.

All in all, with various forms of Marine observation platform technology and the rapid development of equipment and application, such as dissolved oxygen on the key of the ecological environmental water quality parameters of long-term, automatic and continuous monitoring requirements will be more and more, the in situ measuring optical dissolved oxygen and its similar optical sensors in situ calibration method has important significance.

\section{Acknowledgments}

This publication was prepared with the support of the National Key R\&D Program of China (No. 2020ZLHY04), Special Program on Self-Innovation Ability Improvement (Smart Ocean Project) Central Infrastructure Investment Program 2020 (No. Z135060000070) and Qingdao Application Basic Research Plan (No. 19-6-2-26-cg).

\section{References}

[1] Shitanda I, Mori S, Itagaki M. 2011 Screen-printed Dissolved Oxygen Sensor Based on Cerium Oxide-supported Silver Catalyst and Polydimethylsiloxane Film ( Analytical Sciences) 27(10) 1049-1052. 
[2] Martin C S, Dadamos T R L, Teixeira M F S. 2012 Development of an electrochemical sensor for determination of dissolved oxygen by nickel-salen polymeric film modified electrode (Sensors and Actuators B-Chemical) 175 111-117.

[3] LI, FU, YUHONG, et al. 2015 Dissolved oxygen detection by galvanic displacement-induced graphene/silver nanocomposite (Bulletin of Materials Science) 38(3) 611-616.

[4] Moya A, Sowade E, del Campo F J, et al. 2016 All-inkjet-printed dissolved oxygen sensors on flexible plastic substrates (Organic Electronics) 39 168-176.

[5] Lee H J, Kim H M, Park J H, et al. 2017 Fabrication and characterization of micro dissolved oxygen sensor activated on demand using electrolysis (Sensors \& Actuators B Chemical) 241 923-930.

[6] Campo J C, Perez M A, Gonzalez M, et al. 1999 An optrode type sensor to measure dissolved oxygen in water; proceedings of the IEEE Instrumentation \& Measurement Technology Conference F [C].

[7] Shortreed M, Kopelman R, Kuhn M, et al. 1996 Fluorescent Fiber-Optic Calcium Sensor for Physiological Measurements [J]. Analytical Chemistry 68(8) 1414-1418.

[8] Xavier M P, García-Fresnadillo D, Moreno-Bondi M C, et al. 1998 Oxygen Sensing in Nonaqueous Media Using Porous Glass with Covalently Bound Luminescent Ru(II) Complexes (Analytical Chemistry) 70(24) 5184-5189.

[9] Wolfbeis O S. 2002 Fiber-Optic Chemical Sensors and Biosensors (Analytical Chemistry) 74(12) 2663-2677.

[10] Zaitsev N K, Dvorkin V I, Melnikov P V, et al. 2018 A Dissolved Oxygen Analyzer with an Optical Sensor ( Journal of Analytical Chemistry) 73(1) 102-108.

[11] Uchida H, Kawano T, Kaneko I, et al. 2008 In Situ Calibration of Optode-Based Oxygen Sensors ( Journal of Atmospheric and Oceanic Technology) 25(12) 2271-2281.

[12] Takeshita Y, Martz T R, Johnson K S, et al. 2013 A climatology-based quality control procedure for profiling float oxygen data ( Journal of Geophysical Research-Oceans) 118(10) 5640-5650.

[13] Koertzinger A, Schimanski J, Send U. 2005 High Quality Oxygen Measurements from Profiling Floats: A Promising New Technique (Jatmos \& Oceantech) 22(3) 302-308.

[14] D'Asaro E A, McNeil C. 2013 Calibration and Stability of Oxygen Sensors on Autonomous Floats ( Journal of Atmospheric and Oceanic Technology) 30(8) 1896-1906.

[15] Bushinsky S M, Emerson S. 2013 A method for in-situ calibration of Aanderaa oxygen sensors on surface moorings (Marine Chemistry) 155 22-28.

[16] Johnson K S, Plant J N, Riser S C, et al. 2015 Air Oxygen Calibration of Oxygen Optodes on a Profiling Float Array (Journal of Atmospheric and Oceanic Technology) 32(11) 2160-2172.

[17] Nicholson D P, Feen M L. 2017 Air calibration of an oxygen optode on an underwater glider ( Limnology and Oceanography-Methods) 15(5) 495-502. 